\title{
Scenario Development for Safety Training/Education System in Chemical Plant
}

\author{
Atsuko Nakai ${ }^{1}$, Kazuhiko Suzuki ${ }^{1,2}$ \\ ${ }^{1}$ Center for Safe and Disaster-Resistant Society, Okayama University, Okayama, Japan \\ ${ }^{2}$ Graduate School of Natural Science \& Technology, Okayama University, Okayama, Japan \\ Email address: \\ fumoto@safelab.sys.okayama-u.ac.jp (A. Nakai), kazu@sys.okayama-u.ac.jp (K. Suzuki)
}

\section{To cite this article:}

Atsuko Nakai, Kazuhiko Suzuki. Scenario Development for Safety Training/Education System in Chemical Plant. Science Journal of Education. Vol. 3, No. 3, 2015, pp. 68-74. doi: 10.11648/j.sjedu.20150303.14

\begin{abstract}
Safety culture is essentially important in chemical engineering. Because many kinds of hazardous materials are under controlled in chemical facilities. If a severe accident happens, there is a risk for serious damage to employees and the residents of local communities. So our society needs to be assured about the safety and reliability of chemical plants. However the retirement of a skillful worker is advancing in Japan. For this reason, the expert's knowledge and skill are lost, the immature young operator is increasing. As a result operators/workers cannot adequate correspond to abnormal situation in chemical plants. Safety training is important for operators to make a correct decision in emergency. The safety training system will be conveyed based on the training scenario. Therefore in this paper, we propose how to make scenarios for safety training system in chemical plant. The safety awareness of operators will improve by experiential training in case of non-steady situation. The scenarios in the safety training system explain the role-sharing arrangement of workers and plant condition. In order to build a reliable safety culture, mutual understanding and the sharing of safety information are important. Safety training that based on this scenario is useful for the transference of technology and safety knowledge.
\end{abstract}

Keywords: Safety Education, Safety Culture, Scenario Development

\section{Introduction}

In an emergency, chemical plant operators are required to make quick decisions to prevent the expansion of an accident. An untrained operator cannot properly respond to emergencies. Workers' safety awareness can be improved by experiential learning. Education and training about non-standard operations is required for emergency operations. However, reproducing the non-standard conditions of actual equipment or mock-up equipment is not possible because it is too dangerous. Using virtual reality (VR) technology, we can build a virtual chemical plant with lower costs, as compared to a real plant. With the VR plant, operators can experience accidents involving fire and explosions in a virtual space [1]. This paper presents the safety training scenarios for the training system in chemical plants using a virtual plant. Accidents do not always happen the way they are described in manuals, so individuals should be made aware of what to do in the case of an emergency [2]. The proposed scenarios in the safety training system will work to improvements in operators' safety awareness in the event of an unstable situation.

\section{Purpose and Approach}

The safety training system was developed using VR as a training environment. VR is a technology that artificially creates a sense of reality. In a chemical plant, operators must be responsible for their own areas, to ensure the safe operation of all plant components. They are liable for filling two positions: field men and board men. Field men have the role of operating equipment in the field. Board men have the role of controlling refineries from a control center or control room. The scenarios we developed in the safety training system explain the role-sharing arrangement of workers and plant condition. A trainee in this VR system can experience work as a field man or a board man in an environment that is similar to that of an actual chemical plant [1]. The proposed system is composed of a combination of computer graphics and dynamic plant simulator (DS) information. The trainee can feel as if he or she is actually experiencing things in the virtual environment. The DS integrates dynamic simulation environments that reproduce the behaviors of actual plant operations [3]. In this study, a virtual plant works in 
concurrence linked with the DS; as a result, it is possible to reflect on changes in process values corresponding to the passage of time in a VR environment [4]. Operators and workers can learn correct operations through these simulated experiences, to prevent accidents from occurring. The system was developed to consider both field men and board men. A board man trainee inputs the process values in the DS display. The results are then reflected in the virtual plant. A field man trainee next operates the equipment using an avatar in the virtual plant through the virtual plant display. An event progresses qualitatively through the use of animation in the virtual plant and using the process values corresponding to the DS, according to the desired amount of operational change [3] The training system convey based on the training scenarios stored in a behavior scenario database. The training system is constructed using three sub-systems and also consists of two parts: a virtual plant and a dynamic simulator. The biggest difference between web learning and VR environments is that a trainee can reach out into the virtual world and use the VR technology [4] [5]. The safety training system outline is shown in Figure 1.

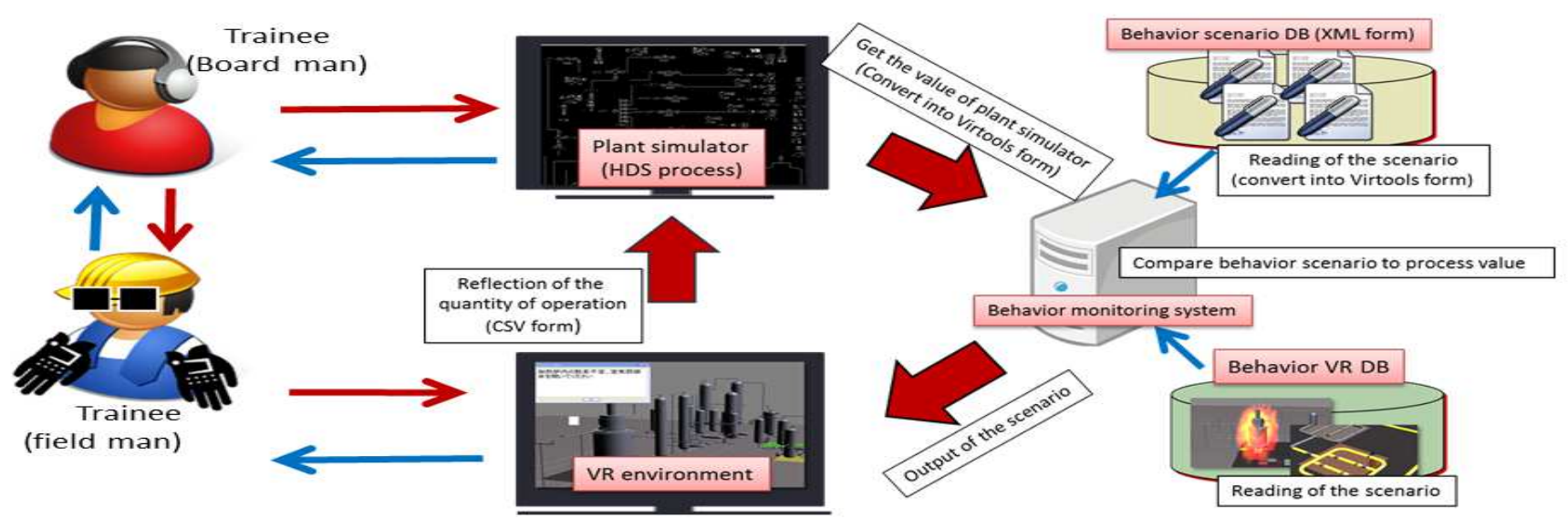

Figure 1. Outline of the safety training system.

\section{Scenario Development for Safety Training System}

\subsection{Behavior Scenario Database and Behavior VR Scene Database}

Safety training for operators using this system is conveyed based on an accident progression scenario. This system linked with the DS. The training in the virtual plant starts with a malfunction. The behavior scenario describes the branch criteria in terms of a threshold, and the event contents are created before the training begins. Figure 2 shows the behavior scenario database. One or more scenarios are stored in it. In the scenario, the branch condition of each piece of plant equipment is based on the process values, such as flow rate, pressure, temperature, etc. A behavior scenario is written using XML. After the divergence, the equipment status is described. It is changed in the VR environment by making the scenario for each piece of equipment fit according to the situation depicted in the simulator. Each piece of equipment has its own behavior protocol. The system also has another database, the "Behavior VR scene database." The VR scene is a combination of the behavior of each piece of equipment, the plant operation scenario, the final scenario, and the results presentation scenario. VR scenes are created along with the training scenario and stored in the database. Training begins with the plant scene. Figure 3 shows the behavior of the VR scenario database.

\subsection{Behavior Monitoring System}

The plant model in a virtual plant and the process values of the DS are linked to a behavior monitoring system. This system reflects the changes in the process values through the passage of time in the VR environment by synchronizing the plant model.

Therefore, the changes in the process values in a real plant are reflected in the virtual plant. The scenario reflection system compares thresholds to write a select scenario and process value changes in the DS. Each process value of the plant simulator is stored in Excel and output as a CSV data form. When the process values meet the branch criteria, the event is progressing based on the qualitatively updated VR scenario and an initial parameter to correspond with it. In the case where a process value meets the branch criteria, the VR scenario and the parameter of the process value are read by the next VR plant and DS parameter. Moreover, the equipment operation is synchronized with the simulator by extracting data about the operation in the VR environment and written to the CSV data for the DS operation through Excel files, as shown in Figure 4. 


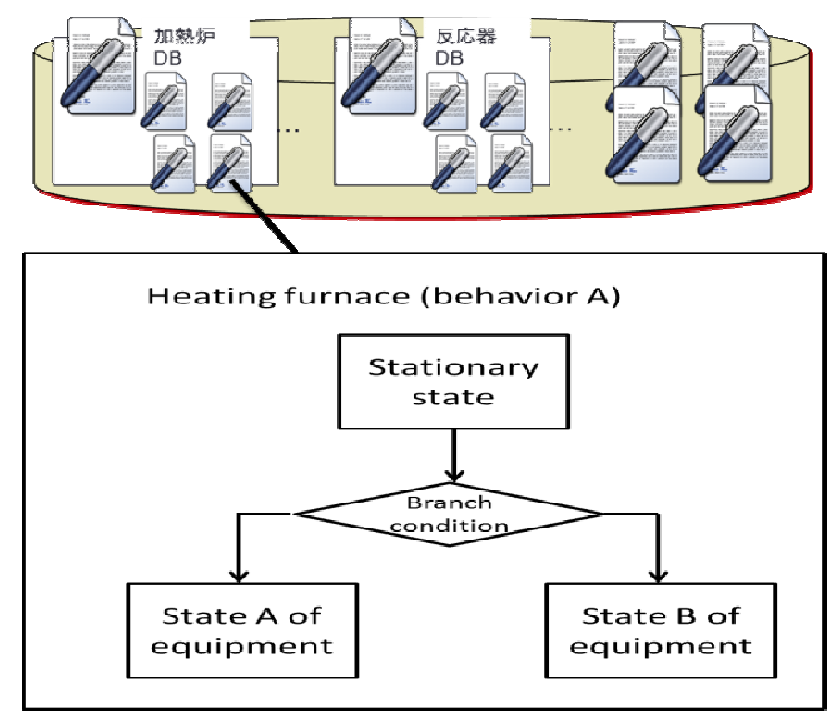

Figure 2. The behavior scenario database.

\subsection{The flow of Training}

Using this system, operators can learn both the board man and field man roles. The training starts with a scene in a virtual plant. The system reads one or more scenarios that describe the branch criteria in terms of the threshold and the event contents from a scenario database. Next, each VR scene is shown, and initial parameters are calculated in advance. The

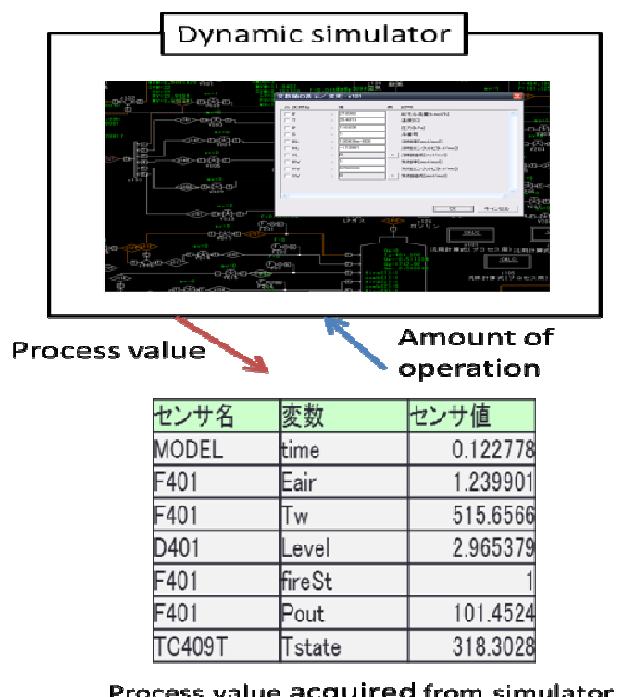

virtual plant model and the process value of the DS are linked by the behavior monitoring system. Therefore, changes in the process values are reflected in the virtual plant. The behavior monitoring system compares the threshold, which leads to a select scenario, and processes the value change of the DS. When the process values meet the branch criteria, the event is progressing and it updates the VR scene and an initial parameter to correspond with it. Figure 5 shows the flow of the training session in the system. This system can show the trainees both a virtual plant and simulator display. When the training starts, the display shows a virtual plant scene. A trainer controls an avatar and makes an operation in the work site.

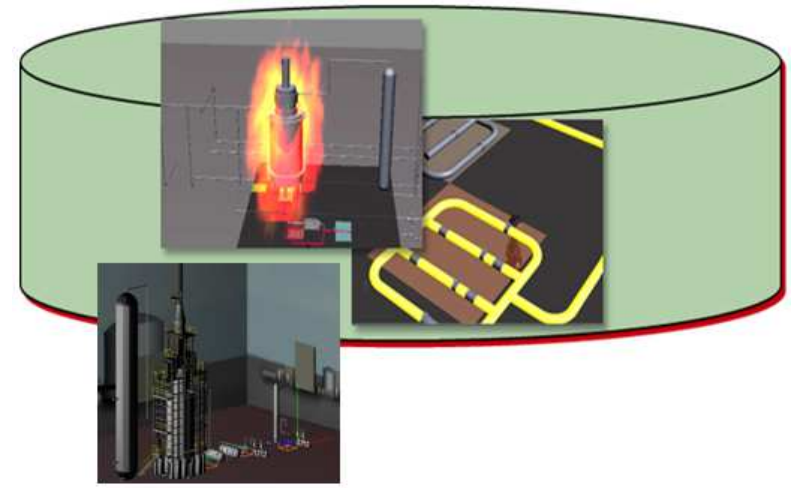

Figure 3. The behavior VR scenario database.

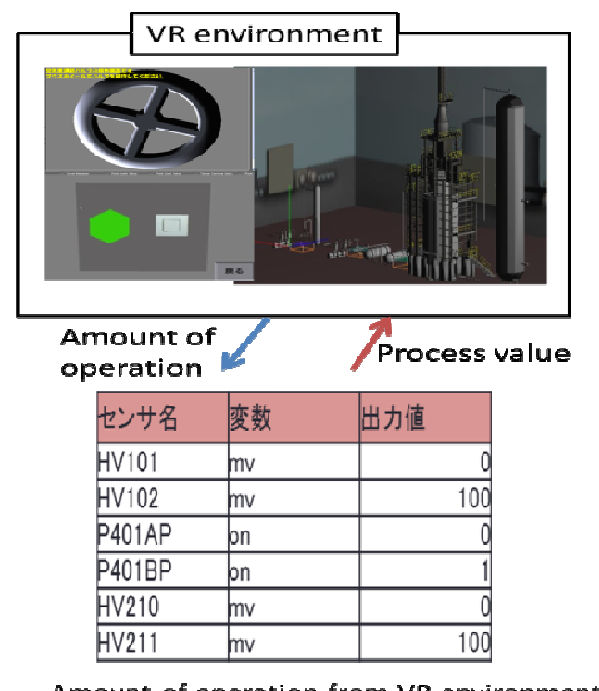

Amount of operation from VR environment

Figure 4. Composition of the behavior monitoring system.

When the avatar approaches the equipment, the operation scene is shown on the display. This training system is updated for each corresponding scene. This system shows the results for each scenario when a trainee is successful in detecting an abnormal situation early and accurately performs the corresponding operation. Conversely, if a trainee fails the operation, the system shows an accident scene. After that, the scene shifts to the results display. The results are shown regarding the assembly operation that the trainer chose. The trainee will then experience an accident like fire or explosion in the virtual plant. This prompts him or her to think about what to do in such an emergency. Figure 6 shows the workflow of a trainee using this system. Safety training using this proposed system is based on a progression through non-standard conditions scenarios. We have developed the training environment for two operators. A trainer can conduct the training session in accordance with the aim of the corresponding operations needed to recover from the accident. 


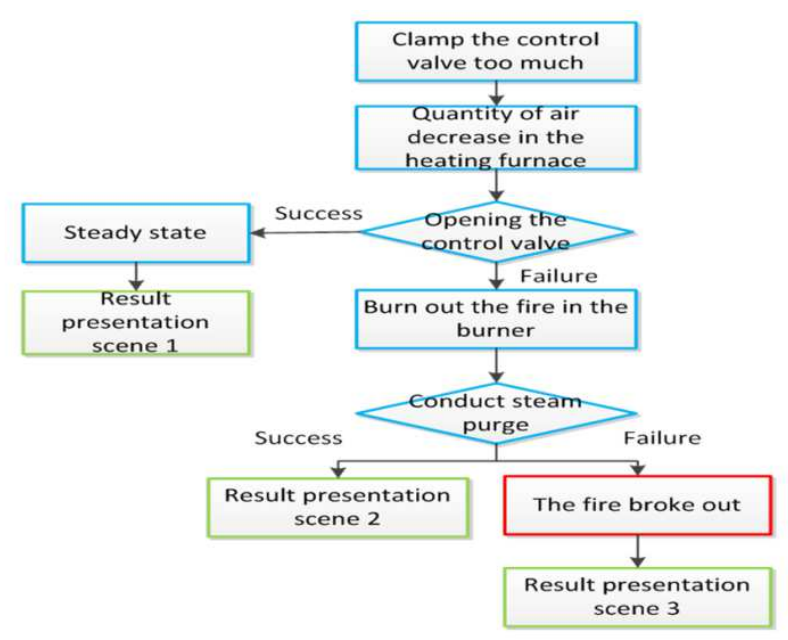

Figure 5. The flow of the training session in the system.

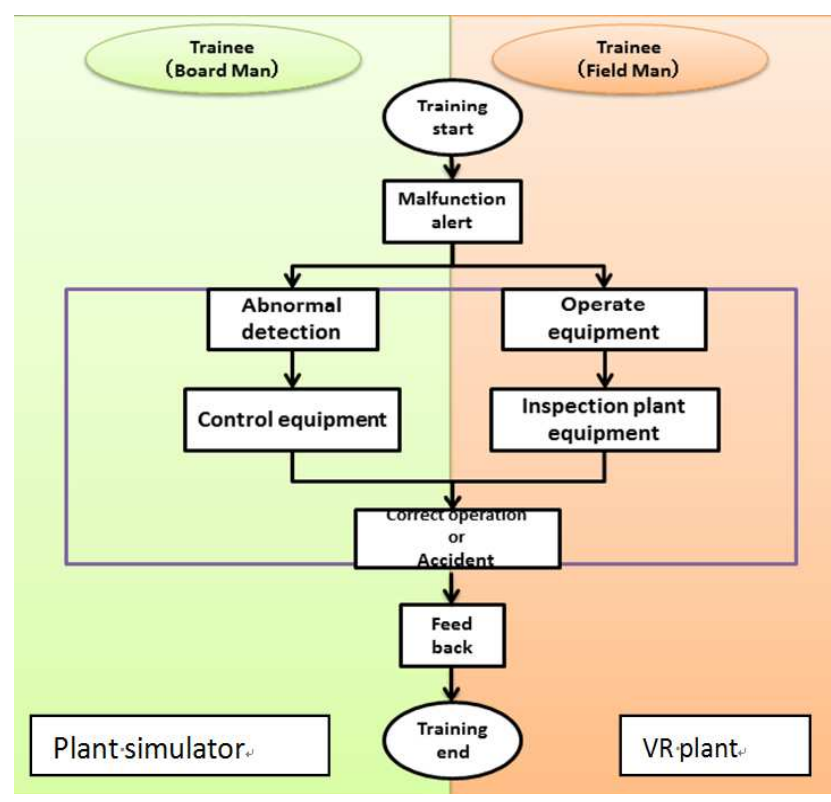

Figure 6. The workflow of the trainee using this system.

\subsection{Non-standard Conditions Scenarios}

Toward more effective training for operators, we have created non-standard conditions scenarios that are based on the cases of potential accidents. The trainer reproduces the circumstances of an accident in the DS in accordance with the scenario. Trainees can learn the correct operations and gain knowledge from this virtual experience [6] [7]. In this training, which involves multitasking performance, communication between trainees is very important, in order to ensure proper operational procedures are followed. Non-standard conditions scenarios are based on past accident reports. There are also some malfunction scenarios in the DS, but standard operating procedures do not cover the operator's performance and communication skills. We assembled our information from accident reports and operations manuals, so that we can recreate non-standard conditions scenarios to program into the computer. The information is kept separate and stored in a database. Figure 7 shows how the non-standard conditions scenarios are kept separate from the rest of the information. The plant conditions are determined by process values in the DS. When a trainee fails to perform the correct operation, the training session is over.

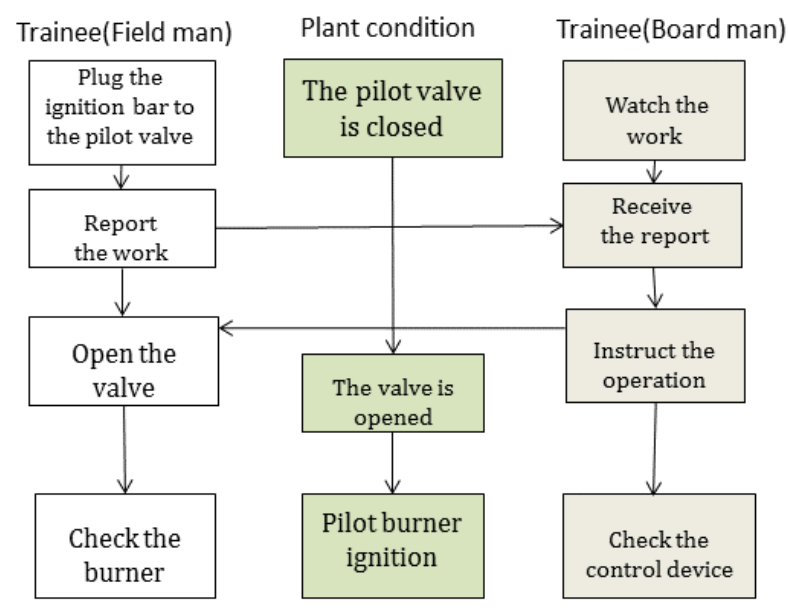

Figure 7. Make the plant condition scenario.

\subsection{Scenarios for the Safety Training System}

In this study, we have developed some scenarios for the safety training/education in chemical plant. The scenarios based on standard operating procedures and malfunction procedure manuals. The purpose of safety training is that the operator can learn the correct operation of the emergency [8] [9]. If the trainees fail the operation in the virtual plant, they can't avoid to experience an accident like fire or explosion. In the standard operating procedures, there have been too much amount of the information described. In the system, it is needed to describe the role of the workers clearly. Accordingly we sorted the information by using the $6 \mathrm{w} 2 \mathrm{~h}$ method. The $6 \mathrm{w}$ means; what, who, when, where, whom, why, and $2 \mathrm{~h}$ means; how, how many. The scenario is described each operation in the tree by dividing information in the standard operating procedures. There are some events in the scenario tree. The branch is made for the trainee success or fail the operation. Figure 8 shows the basic model of the scenario development.

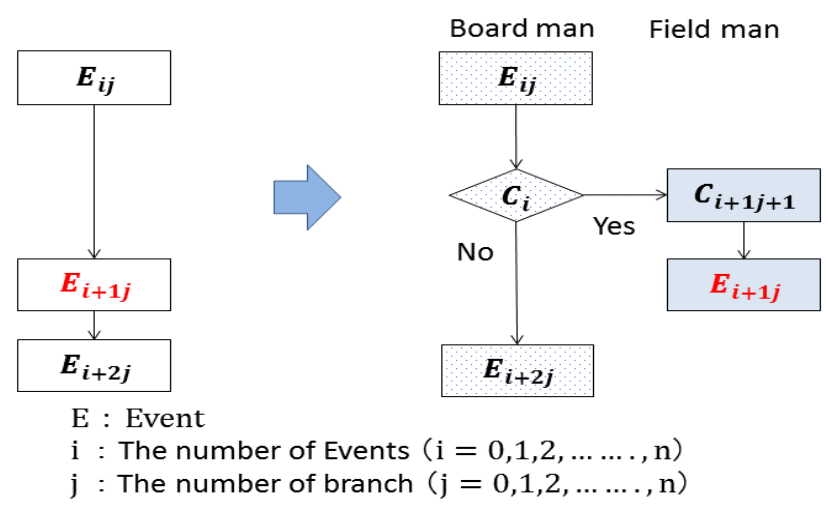

Figure 8. The basic model of the scenario. 


\section{Case Study}

\subsection{Application of the Hydrodesulfurization Process}

This system is supposed to simulate the hydrodesulfurization process production plant model. This process is the reaction that comes with hydro-gen separation and the removing the sulfur content in petroleum using the metal catalysts of nickel, cobalt, molybdenum, etc. under high temperature and high pressure, and producing products with low kerosene and sulfur content, and light oil. The outline of this process is shown in Figure 9

\subsection{Making the Training Scenario}

In this paper, we created a scenario involving VGO feed trouble. The prerequisites for carrying out the HDS training operations are as follows:

1. The time to activate a spare pump starts when the pump is stopped, in order to close the flow time of the real device. This takes about 3 minutes.

2. "Communication," "reporting," etc. should reflect the training of operators. They should protect what has been done within the department or company.

Corresponding operations are as follows:

1. The field workers need to switch a device in the circulation.

2. It is necessary to pay attention to the rising temperature of the furnace, due to the temperature rise of the reactor.

3. A board man should operate the DCS using caution, monitoring any change in the temperature of the intake and outflow of the furnace.

4. A field man should work to switch to a device in the circulation in the field.

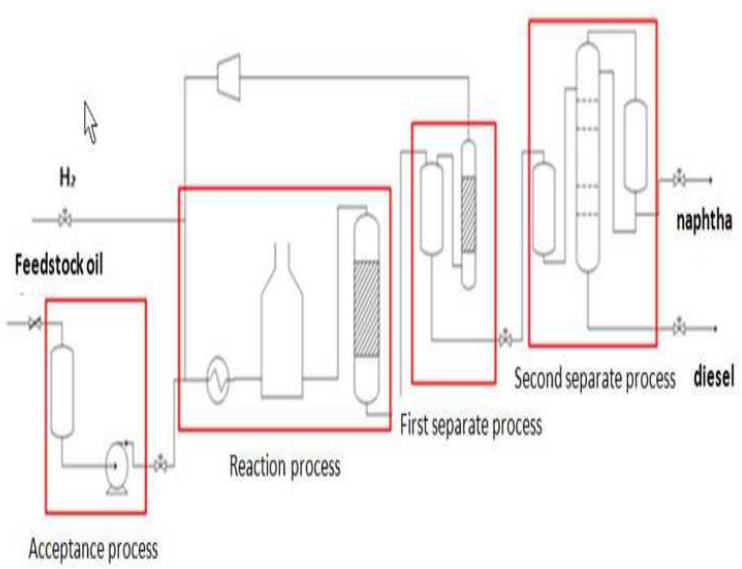

Figure 9. Outline of the hydrodesulfurization process.

The training scenario is created based on these parameters. This operation calls for both operations, a board man, and a field man. In this system, the process value is linked with the plant model in the VR environment by the behavior-monitoring system of the DS. The system compares the branch conditions in the scenario using the process values stored in the array of the VR plant and outputs a corresponding scenario. The purpose of this training is threefold. A branch condition in the scenario is created for the purposes of training.

1. Field Man: Switch promptly to circulation (valve operation, communicate with the valve adjustment board man).

2. Board Man: Narrow the furnace intake flow rate, adjust the outflow temperature (give operation instructions to the field man, confirm the operation, communication, etc.).

3. Board man: Pay attention to the temperature change of the heating furnace exit (external supply VGO is close to $190^{\circ} \mathrm{C}$, circulation DVGO is about $65^{\circ} \mathrm{C}$ ).

In this case, workers should suppress the temperature rise in the heating furnace, to prevent an accident. The training scenario (for non-standard conditions scenarios) is shown in Figure 10. The information from the malfunction correction operations manual has been assembled related to trainee behavior or plant conditions. Therefore, the branch conditions are settled for the purpose of training. Plant conditions are stored in the system as a behavior scenario in the behavior scenario database.

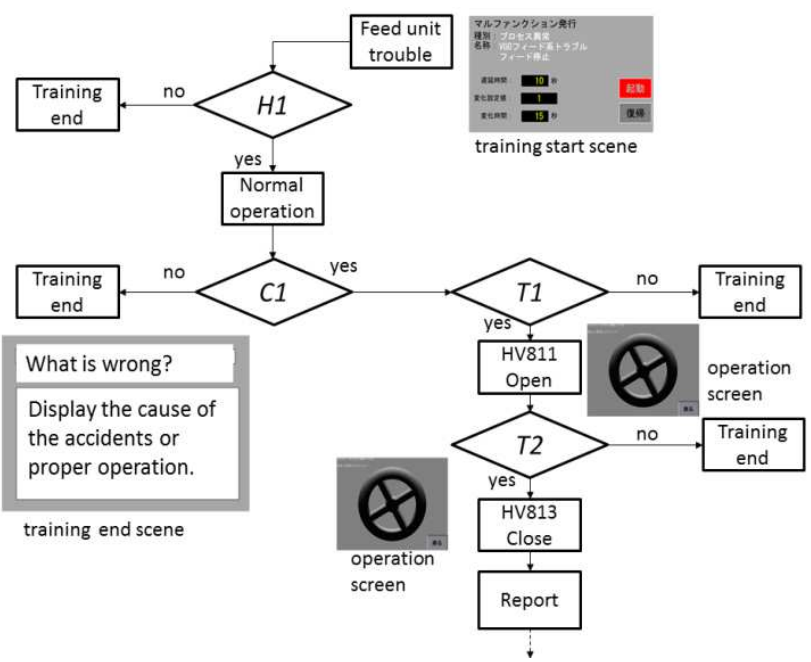

Figure 10. The training scenario.

The branch conditions are described as part of the training scenario referring to equipment operations. Suspending the DS can stop the simulation. Thus, the event is divided by the threshold of the process value. The event can be continuously shown by creating a simulation based on the initial parameters of the process value, which differs with each event.

\subsection{Training Based on the Scenario}

Training based on the scenario is applied to our proposed system using a virtual plant-linked dynamic simulator. Figure 11 shows the safety training system display for trainees. The left side display shows the virtual plant for a field man. The right side display shows the DCS for a board man. The trainer can check both displays and the center DS display throughout the training process. The scenarios in the system are based on the DS malfunction operating procedures. In real chemical 
plant we have many situation for safety training. The development basic scenario model can apply another

malfunction procedures.

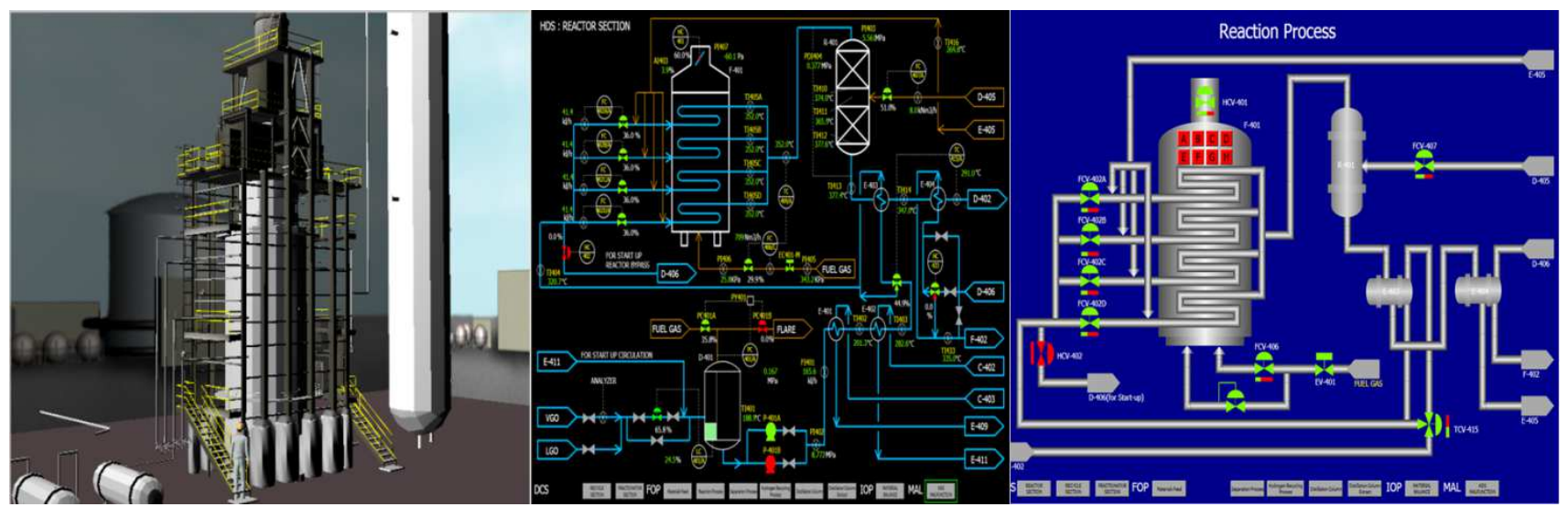

Figure 11. The safety training system using virtual plant-linked dynamic simulator.

\section{Discussion}

The experience-based safety training and safety education is efficiently to improve the safety environment for employees and local residents near the plant [1] [10]. By using VR technology, the costs and location problems associated with non-standard events training can be solved [11]. Trainees can learn safely about the operations they need to perform in the event of a problem in the plant. This paper shows that the scenarios can work in the safety training system and they are useful for safety education. The scenario that we developed can using for another situation. It is based on the standard operating procedures. We sort out the information considering an accident case. The scenario can clear the operation/procedure problem. In the future safety education, we need more training/education scenario. In addition it is required considering human error and temporal progress.

\section{Conclusion}

In this study, we have developed scenarios for the safety training system in chemical plant. The scenarios are development for a cooperative working environment similar to what they would encounter in a real chemical plant. At the chemical plant, operators are required to possess accurate knowledge and be skillful enough to prevent accidents [5] [12]. Through training using the scenarios, a trainee can learned how to prevent the hazardous conditions. But in the future, it is necessary to develop a scenario that takes into account the timing of the operation. In addition the completely automatic scenario making system will required to correspond more situations for safety training.

In chemical plants in Japan, there have been rapid generational changes in the workers. Inheriting technology and past accomplishments in a chemical plant is essential for enhancing competitive production. Safety education educates individuals on various safety practices at work, at school, on the road and in daily life. For our future generations, we need a steady and sustainable culture of safety in the field of chemical engineering. By using the scenarios for safety training, we will lead to improvements in safety awareness during day-to-day operations, due to the increases in skill, sensibility, knowledge, and overall competence it provides.

\section{References}

[1] Nakai, A., Kaihata, Y., Suzuki, K. 2014. "The Experience-Based Safety Training System Using $\mathrm{Vr}$ Technology for Chemical Plant", International Journal of Advanced Computer Science and Applications (IJACSA), Vol.5, No.11, pp.63-67

[2] Jürgen, S. 2013. "Process and Plant Safety - Research \& Education Strategy to Keep Long Term Competences", Chemical Engineering Transactions, vol.31.

[3] Fukano, G., Yokoyama, K., Yahata, Y. 2013. "MIRROR PLANT On-line Plant Simulator and its Applications", Yokogawa Technical Report English Edition, vol.56 No.1, pp. $11-14$.

[4] Yamamoto, K., Nakai, A., Suzuki, K. 2013. "Development of Experienced-based Training System combined with Process Dynamic Simulation” Asia Pacific Symposium on Safety 2013.

[5] Invensys Process Systems. 2012. "U.S. Department of Energy Deploys Virtual Reality Training Solution from Invensys Operations Management"

[6] Norton, C., Cameron, I., Crosthwaite, C., Balliu, N., Tade, M., Shallcross, D., Hoadley, A., Barton, G., Kavanagh, J. 2008. "Development and deployment of an immersive learning environment for enhancing process systems engineering concepts", Education for Chemical Engineers, vol. 3, 2, December pp.75-83.

[7] Schofield, D. 2012. "Mass Effect: A Chemical Engineering Education Application of Virtual Reality Simulator Technology", MERLOT Journal of Online Learning and Teaching vol. 8, No. 1, March.

[8] Chen Xi, Wu, H., Joher, A., Kirsch, L., Luo, C. 2009. "3-D Virtual Reality for Education, Training and Improved Human”, ANS NPIC HMIT 2009 Topical Meeting. 
[9] Stephan, E., Avestar, Z.2013“Advanced Virtual Energy Simulation. R\&D087".

[10] Lee, Y., Kim, Ji., Kim, Ju., Kim, Eu., Kim, Yp. 2010. "Development of a web-based 3D virtual reality program for hydrogen station"International Journal of Hydrogen Energy,Vol35, pp.2112-2118.

[11] Ponder, M., Herbelin, B., Molet, T., Schertenlieb, S. 2003.
"Immersive VR Decision Training - Telling Interactive Stories Featuring Advanced Virtual Human Simulation Technologies" The Eurographics Association 2003.

[12] Manca, D., Brambilla, S., Colombo, S. 2013. "Bridging between Virtual Reality and accident simulation for training of process-industry operators", Advances in Engineering Software, Volume 55, January, Pages 1-9. 